\title{
A modified chief complaint-based cardiac triage strategy for reducing delays in the management of patients with ST-elevation myocardial infarction
}

Hung-Yuan Su

E-Da Hospital

Jen-Long Tsai

E-Da Hospital

Yin-Chou Hsu

E-Da Hospital

Kuo-Hsin Lee

E-Da Hospital

Chao-Sheng Chang

E-Da Hospital

Cheuk-Kwan Sun

E-Da Hospital

Yu-Han Wang

E-Da Hospital

Chih-Wei Hsu (D saab30002000@gmail.com )

E-Da Hospital

\section{Research Article}

Keywords: door-to-balloon time, door-to-ECG time, ST-elevation myocardial infarction, percutaneous coronary intervention

Posted Date: January 13th, 2021

DOI: https://doi.org/10.21203/rs.3.rs-141934/v1

License: (9) This work is licensed under a Creative Commons Attribution 4.0 International License.

Read Full License

Version of Record: A version of this preprint was published at Scientific Reports on March 18th, 2021. See the published version at https://doi.org/10.1038/s41598-021-86013-8. 


\section{Abstract}

Timely performing electrocardiography (ECG) is crucial for early detection of ST-elevation myocardial infarction (STEMI). For shortening door-to-ECG time, a chief complaint-based "cardiac triage" protocol comprising (1) raising alert among medical staff with bedside triage tags, and (2) immediate bedside ECG after focused history-taking was implemented at the emergency department (ED) in a single tertiary referral center. All patients diagnosed with STEMI visiting the ED between November 2017 and January 2020 were retrospectively reviewed to investigate the effectiveness of strategy before and after implantation. Analysis of a total of 117 ED patients with STEMI (pre-intervention group, $n=57$; postintervention group, $n=60$ ) showed significant overall improvements in median door-to-ECG time from 5 to 4 minutes $(p=0.02)$, achievement rate of door-to-ECG time $<10$ minutes from $45-57 \%(p=0.01)$, median door-to-balloon time from 81 to 70 minutes $(p<0.01)$. Significant trends of increase in achievement rates for door-to-ECG and door-to-balloon times ( $p=0.01$ and $p=0.006$, respectively) was noticed after strategy implementation. The incidence of door-to-ECG time $>10$ minutes for those with initially underestimated disease severity was also reduced from $90-10 \%(p<0.01)$. In conclusion, a chief complaint-based "cardiac triage" strategy successfully improved the quality of emergency care for STEMI patients through reducing delays in diagnosis and treatment.

\section{Introduction}

Primary percutaneous coronary intervention $(\mathrm{PCl})$ is the gold-standard treatment for patients with STelevation myocardial infarction (STEMI) ${ }^{1,2}$. Timely coronary artery reperfusion after arriving at emergency department (ED) is important for reducing mortality and morbidity rates for patients with STEMI ${ }^{3-5}$. Doorto-Balloon Alliance has chosen key strategies for improving door-to-balloon (DTB) time, which included the activation of the catheterization laboratory with a single call by emergency physicians, completion of $\mathrm{PCl}$ team preparation within 20 to 30 minutes after the call, rapid data feedback, adoption of a teambased approach, and administrative support ${ }^{6,7}$. Additionally, the American College of Cardiology and American Heart Association (ACC/AHA) has also recommended the target times of door-to-ECG (DTE) within 10 minutes and DTB within 90 minutes, respectively, which have become the benchmark for the management of acute coronary syndrome worldwide ${ }^{1}$.

Rapid performance of electrocardiography (ECG) for STEMI identification is crucial to achieving coronary artery reperfusion. Some studies have shown that improving DTE time can shorten DTB time ${ }^{8-10}$. Although a previous large-scale multicenter study has demonstrated no significant reduction in 30-day inhospital mortality rate for STEMI patients achieving the target time of DTB within 90 minutes, improving DTE and DTB times should still be persistently emphasized owing to the potential benefits of long-term reduction in mortality, improvement in left ventricular function, and decreasing the number of admissions for heart failure ${ }^{11}$. On the other hand, only 20 to 30 percent of patients with cardiac ischemic symptoms achieved the target time of DTE within 10 minutes $^{8,12}$ so that several quality improvement programs had been proposed for shortening the DTE time. Among them, triage ECG, which is the acquisition of ECG 
during triage before history-taking by emergency physicians, is widely implemented in the ED setting ${ }^{4,10,13-15}$. However, the downside of triage ECG is that, in addition to the need for setting up ECG equipment, technicians and space in the triage area, indiscriminate ECG for patients with suspected STEMI is not cost-effective and can be time-consuming so that the care for other patients could be delayed $^{16}$. To address this issue, Coyne et al. created a "cardiac triage" designation to incorporate triage ECG into an improved patient disposition during the triage process, which has been reported to shorten the DTE time ${ }^{17}$. Although this combined approach was successful in reducing DTE time, some concerns, such as the increased workload among medical staff, interruption and distraction at work as well as a high cost of employing specialized personnel with a low yield of STEMI identification, were raised in a few studies ${ }^{16,18}$.

Therefore, our study modified the combined process to evaluate if cardiac triage alone can improve ECG performance in ED by hanging a red warning tag on the bedside of patients who were suspected of having STEMI by the triage nurse and placing their medical charts in a designated area to expedite subsequent managements, including prompt history-taking by emergency physicians and ECG. The current study aimed at assessment of DTE and DTB time and related factors associated with delayed ECG acquisition.

\section{Methods}

\section{Study design and patient population}

The current study was conducted in a 1,251-bed tertiary referral center that had 66,000 emergency visits per year. From November 2017 to January 2020, the electronic medical records of all adult patients ( $\geqq$ 18 years old) who were diagnosed with STEMI in ED and subsequently received primary $\mathrm{PCl}$ in the ED were retrospectively reviewed by a STEMI quality control team every month as a standard procedure of a medical quality improvement program of the institute. The team, which comprised cardiologists, emergency physicians, triage nurses, and quality control specialists, was responsible for monitoring the changes in quality indicators related to STEMI management including DTE time and DTB time as well as implementing appropriate improvement strategies. As a novel quality improvement program, a cardiac triage strategy was introduced in December 2018. Patients who had been diagnosed with STEMI before our ED arrival and/or those who had received resuscitation before ECG acquisition were excluded.

\section{Pre-intervention management}

During the pre-interventional period from November 2017 to November 2018, patients who visited ED were directed to the triage area where they were classified in terms of disease severity and chief complaint into five categories according to the Taiwan Triage and Acuity Scale (TTAS), which was modified from the Canadian Triage and Acuity Scale and has been officially adopted by the Taiwanese emergency health care system since the year 2010: Level I, resuscitation; level II, emergent; level III, urgent; level IV, less urgent; and level V, non-urgent ${ }^{19}$. During this process, patients belonging to level I, II, III, IV, and 
$V$ should be evaluated by emergency physicians or nurse practitioners immediately, $10 \mathrm{~min}, 30 \mathrm{~min}$, $60 \mathrm{~min}$, and $120 \mathrm{~min}$, respectively. Because the triage system did not specifically identify patients with potential ischemic cardiac problems, those presenting with typical cardiac symptoms (e.g., chest pain) as well as those having high cardiovascular risks (i.e., age $>50$, diagnosis of diabetes) with atypical manifestations (i.e., epigastralgia, nausea, dyspnea, diaphoresis) according to the AHA emergency department screening criteria ${ }^{20}$ had to follow the above management time frame according to the triage classification until the emergency physician identified STEMI and activated the "Code STEMI" to notify cardiologists for evaluation and preparation for PCl. On encountering patients with confirmed STEMI, the emergency physicians were required to complete a DTB checklist summarizing the timing of every step of management (e.g., ECG, consultation with cardiologist) between the time when the patients arrived in ED and the time when they received coronary intervention (Fig. 1). All data were recorded in an electronic database.

\section{Pilot study period: timing and procedures}

In one month's period (December 2018), in addition to the five-level triage system, a Cardiac Triage program aiming at prioritizing the management of patients with STEMI was introduced in our emergency care system in an attempt to reduce the DTE time. The new strategy involved two key steps to expedite STEMI diagnosis and management (Fig. 2). First, patients who visited ED were directed to the triage area where the triage nurse would immediately identify possible ischemic cardiac symptoms (e.g., chest pain) as well as atypical presentations in those with high cardiovascular risk ${ }^{20}$ and label them with a red tag and place the medical record in a designated area for immediate management by the emergency physician. Second, history-taking focusing on the possibility of a STEMI was performed by the emergency physician who ordered immediate 12-lead ECG for patients with suspected STEMI and activated the "Code STEMI" for confirmed cases. At the end of one month, a meeting was held for addressing all issues arising from program implementation to ensure adequate communication and satisfactory problem-shooting between the STEMI quality control team and the frontline emergency physicians and nursing staff. Following confirmation of the feasibility of the program, the new strategy was officially implemented from January 2019.

\section{Post-intervention follow-up}

During the post-intervention period (January 2019 to January 2020), concomitant implementation of the five-class triage system and the Cardiac Triage program had become a routine. Data were continually being recorded electronically on the DTB checklist (Fig. 1) for review. Monthly meetings were held to identify unforeseen problems with patient management and data collection during the process of cardiac triage.

\section{Outcomes and definitions}


Data collection was completed by the end of January 2020 when outcomes of the strategy was assessed by the quality control team through analyzing and comparing the data acquired before and after implementation of the Cardiac Triage program. Data during the intervention period (December 2018) was excluded. The primary outcome of the study was the median DTE time and the achievement rate of DTE time $<10$ minutes, while the secondary outcome was the median DTB time and the achievement rate of DTB time $<90$ minutes as well as in-hospital mortality rate. The achievement rates of DTE time $<10$ minutes and DTB time $<90$ minutes were compared before and after implementation of the cardiac triage program by assessing the changes in the mean achievement rates every three months.

Door time, ECG time and balloon time were defined as the times of registration at ED reception, completion of ECG, and first balloon inflation in culprit lesion, respectively ${ }^{21}$. Daytime and nighttime were defined as the periods from 07:01 to 17:00 and from 17:01 to 07:00, respectively. Weekdays and weekend were defined as from Monday morning to Friday night and from Saturday morning to Sunday night, respectively. For triage categories, high and low triage levels were defined as category I, II and category III, IV, V, respectively.

\section{Statistical analysis}

All data were analyzed by using SPSS version 22 (SPSS Inc, Chicago, IL). Mean values and median values were expressed as mean $\pm S D$ and median (IQR) respectively. Student's t-test and Chi-squared test were used for determining the significance of difference among continuous and categorical variables, respectively. Fisher's exact test was used to identify risk factors related to delayed ECG acquirement (i.e., $>10$ minutes after ED arrival) before and after interventions. Paired sample t-test was used for determining the significance of changes in the achievement rates of DTE time $<10$ minutes and DTB time $<90$ minutes after implementation of the cardiac triage strategy. A two-tailed $p$ value of less than 0.05 was considered statistically significant.

\section{Ethics committee approval}

Ethics approval for this study was granted by the Institutional Review Board of the E-DA Hospital (EMRP109-026) and the need of informed consents was waived because of the retrospective nature of the present study. All methods were carried out in accordance with relevant guidelines and regulations.

\section{Results}

\section{Study population}

Between November 2017 and January 2020, there were a total of 136,402 ED visits and 12,058 ECG examinations. Among them, 6,063 visits (8.83\%) involved ECG in pre-intervention period and 5,995 visits (8.84\%) in post-intervention period. Of the 193 patients with the diagnosis of STEMI, 66 had been diagnosed with STEMI before arrival at our ED and the other four had received resuscitation before ECG acquisition. Additionally, after excluding six more patients diagnosed with STEMI during the one-month pilot study period (December 2018), a total of 117 patients were enrolled into this study, including 57 in 
the pre-intervention group and 60 in the post-intervention group. A review of the characteristics of our patients with STEMI during the study period demonstrated no significant difference in age, gender, triage category, means of ED arrival, visiting time, typical angina presentations, and comorbidities between the pre- and post-intervention groups (Table 1). 
Baseline characteristics of STEMI patients $(n=117)$

\begin{tabular}{|llll|}
\hline Characteristics & $\begin{array}{l}\text { Pre-intervention group }(\mathrm{n}= \\
\text { 57) }\end{array}$ & $\begin{array}{l}\text { Post-intervention group }(\mathrm{n}= \\
\mathbf{6 0})\end{array}$ & $\begin{array}{l}\boldsymbol{p} \\
\text { value }\end{array}$ \\
\hline Age, $\mathrm{y}$, mean \pm SD & $62.5 \pm 13.9$ & $62.2 \pm 11.1$ & 0.91 \\
\hline Male, $\mathrm{n}(\%)$ & $50(87.7)$ & $52(86.7)$ & 1.00 \\
${ }^{\dagger}$ Triage Category, $\mathrm{n}(\%)$ & $13(22.8)$ & $8(13.3)$ & 0.43 \\
Category 1 & $34(59.6)$ & $42(70.0)$ & \\
Category 2 & $10(17.5)$ & $10(16.7)$ & \\
Category 3 & 0 & 0 & 0.12 \\
Category 4 & 0 & 0 & \\
Category 5 & & & \\
\hline Means of ED arrival, $\mathrm{n}(\%)$ & & $34(56.7)$ & \\
\hline Walk-in & $41(71.9)$ & $26(43.3)$ & \\
\hline $\begin{array}{l}\text { Emergency medical } \\
\text { service }\end{array}$ & $16(28.1)$ & $27(60.0)$ & \\
\hline ED time, $\mathrm{n}$ (\%) & $34(52.6)$ & & \\
Daytime & & & \\
\hline
\end{tabular}

STEMIST-elevation myocardial infarction, ED emergency department.

†Triage categories according to the Taiwan Triage and Acuity Scale (TTAS), a modification of the Canadian Triage and Acuity Scale 


\begin{tabular}{|c|c|c|c|}
\hline Characteristics & $\begin{array}{l}\text { Pre-intervention group }(n= \\
57)\end{array}$ & $\begin{array}{l}\text { Post-intervention group }(n= \\
60)\end{array}$ & $\begin{array}{l}p \\
\text { value }\end{array}$ \\
\hline Nighttime & $23(47.4)$ & $33(40.0)$ & 0.46 \\
\hline ED day, n (\%) & $30(52.6)$ & $36(60.0)$ & 0.39 \\
\hline Weekday & $27(47.4)$ & $24(40.0)$ & 0.71 \\
\hline Weekend & $49(86.0)$ & $56(93.3)$ & 0.71 \\
\hline Chest pain, n (\%) & $25(43.9)$ & $24(40.0)$ & 0.70 \\
\hline Comorbidities, n (\%) & $31(54.4)$ & 35 (58.3) & 1.00 \\
\hline Diabetes Mellitus & $37(64.9)$ & $41(68.3)$ & 0.57 \\
\hline Hypertension & $4(.7 .0)$ & $4(.6 .7)$ & \\
\hline Dyslipidemia & $20(35.1)$ & $.25(41.7)$ & \\
\hline \multicolumn{4}{|l|}{$\begin{array}{l}\text { Old cerebrovascular } \\
\text { accident }\end{array}$} \\
\hline \multicolumn{4}{|c|}{ Chronic kidney disease } \\
\hline \multicolumn{4}{|c|}{ STEMIST-elevation myocardial infarction, ED emergency department. } \\
\hline \multicolumn{4}{|c|}{$\begin{array}{l}\text { †Triage categories according to the Taiwan Triage and Acuity Scale (TTAS), a modification of the } \\
\text { Canadian Triage and Acuity Scale }\end{array}$} \\
\hline
\end{tabular}

\section{Primary outcomes after intervention}

The DTE time was significantly shorter after intervention than that before intervention (4 minutes vs. 5 minutes, $p=0.02$ ) (Table 2). In addition, there was a higher proportion of patients with DTE $<10$ minutes in the post-intervention group compared with that in the pre-intervention group $(95.0 \%$ vs. $78.9 \%, p=0.01$, respectively). Comparison of the achievement rate of DTE $<10$ minutes before and after implementation of the cardiac triage program showed a significant trend of increase after initiation of the strategy $(p=$ 0.01) (Fig. 3). 
Table 2

Comparison of outcomes among STEMI patients before and after intervention $(n=117)$

\begin{tabular}{|c|c|c|c|}
\hline Variables & $\begin{array}{l}\text { Pre-intervention group }(\mathrm{n} \\
=57)\end{array}$ & $\begin{array}{l}\text { Post-intervention group }(\mathrm{n} \\
=60)\end{array}$ & $\begin{array}{l}p \\
\text { value }\end{array}$ \\
\hline $\begin{array}{l}\text { Door to ECG time (min), median } \\
\text { (IQR) }\end{array}$ & $5.0(1.5-8.0)$ & $.4 .0(1.0-5.0)$ & $0.02^{*}$ \\
\hline Door to ECG time $<10 \mathrm{~min}, \mathrm{n}(\%)$ & $.45(78.9)$ & $57(95.0)$ & $0.01^{*}$ \\
\hline $\begin{array}{l}\text { Door to Balloon time (min), } \\
\text { median (IQR) }\end{array}$ & $81.0(70.5-91.5)$ & $.70 .0(53.3-84.0)$ & $\begin{array}{l}<.01^{\star \star} \\
0\end{array}$ \\
\hline $\begin{array}{l}\text { Door to Balloon time }<90 \text { min, } n \\
(\%)\end{array}$ & $39(68.4)$ & 50 (83.3) & 0.08 \\
\hline \multirow{2}{*}{$\begin{array}{l}\text { Length of stay (day), median } \\
\text { (IQR) }\end{array}$} & $6(5-9)$ & $6(5-7)$ & 0.73 \\
\hline & $3(3-4)$ & $3(3-4)$ & 0.71 \\
\hline ICU stay (day), median (IQR) & $2(3.5)$ & $2(3.3)$ & 1.00 \\
\hline \multicolumn{4}{|c|}{$\begin{array}{l}\text { STEMIST-elevation myocardial infarction, ECG electrocardiogram, IQR interquartile range, ICU } \\
\text { intensive care unit. }\end{array}$} \\
\hline \multicolumn{3}{|l|}{$* p<0.05, * \star p<0.01$} & \\
\hline
\end{tabular}

\section{Secondary outcomes after intervention}

Consistent with the change in DTE time, the DTB time was significantly shorter after intervention compared to that before intervention (70 minutes vs. 81 minutes, $p<0.01$, respectively) (Table 2). Besides, there was a higher proportion of patients achieving DTB time $<90$ minutes in the post-intervention group than that in the pre-intervention group ( $83.3 \%$ vs. $68.4 \%, p=0.08$, respectively) despite the lack of statistical significance. On the other hand, there was a significant trend of increase in achievement rate of DTB time $<90$ minutes after implementation of the cardiac triage the strategy $(p<0.006)$ (Fig. 3). However, there was no significant difference in the length of hospital stay, intensive care unit (ICU) stay, and in-hospital mortality between the pre- and post-intervention groups.

\section{Effectiveness of interventions for improving DTE times}

Our literature search identified four reported contributors to DTE delays, including STEMI without chest pain, underestimated disease severity (i.e., initially low triage levels of III - V), walk-in patient, and female gender $^{8-10,15,22}$. Therefore, the four factors were used for evaluating the effectiveness of the program for improving DTE times in STEMI patients. There were a total of 15 patients with DTE exceeding 10 minutes in the present study, including 12 in pre-intervention group and three in post-intervention group. A low triage level was found to be the most significant predictor of outcome improvement after program intervention because the rate of DTE $>10$ minutes decreased drastically from $90-10 \%$ after strategy implementation $(p<0.01)$. On the other hands, despite decreases in the rate of DTE > 10 minutes were 
also noted in the means of ED arrival (i.e., walk-in) and the female gender, both failed to reach statistical significance ( $p=0.08$ and 0.62 , respectively). In addition, there was no significant impact of STEMI without chest pain on the effectiveness of the cardiac triage program (Table 3 ).

Table 3. Comparison of factors associated with ECG >10 minutes between pre- and post-intervention groups

\begin{tabular}{|lcll|}
\hline Reasons for ECG > 10 min & $\begin{array}{l}\text { Pre-intervention group } \\
{ }^{\dagger} \mathrm{N} 1 / \mathrm{N} 2(\%)\end{array}$ & $\begin{array}{l}\text { Post-intervention group } \\
{ }^{\dagger} \mathrm{N} 1 / \mathrm{N} 2(\%)\end{array}$ & $p$ value \\
\hline STEMI without chest pain & $3 / 800(37.5 \%)$ & $2 / 400(50.0 \%)$ & .1 .00 \\
\hline Low triage level & $9 / 100(90.0 \%)$ & $1 / 100(10.0 \%)$ & $<0.01^{*}$ \\
\hline Walk-in patient & $12 / 410(29.2 \%)$ & $3 / 280(10.7 \%)$ & 00.08 \\
\hline Female gender & $4 / 700(57.1 \%)$ & $3 / 800(37.5 \%)$ & 00.62 \\
\hline
\end{tabular}

ECG electrocardiogram, STEMIST-elevation myocardial infarction

${ }^{\dagger} \mathrm{N} 1$ represents number of STEMI patients with door-to-ECG $>10$ minutes and N2

represents total numbers of STEMI patients.

* $p<0.05$

\section{Discussion}

The current study demonstrated that implementation of a modified cardiac triage protocol for early identification and treatment of patients with STEMI in the emergency care setting could significantly shorten the median DTE time and increase the achievement rate of DTE time less than 10 minutes. Moreover, median DTB time was also significantly reduced. Further investigation also revealed a significant reduction in the incidence of DTE time over 10 minutes among patients belonging to a low triage category (i.e., III, IV, or V) after intervention.

DTB is a survival chain comprising early ECG with prompt interpretation, early catheterization lab activation, an expedited response to activation, and rapid reperfusion ${ }^{17}$. Although multiple factors would affect DTB time, a previous study has shown a stronger association of DTB time with door-to-activation time compared to that with activation-to-laboratory and laboratory-to-balloon times ${ }^{5}$. Timely ECG is crucial to the identification of patients with STEMI for prompt primary PCI. The American Heart Association (ACC/AHA) management guideline for patients presenting with symptoms of cardiac ischemia has indicated a DTB time of less than 10 minutes as a standard for acceptable emergency medical practice ${ }^{1}$. Hence, various efforts have been made to shorten the DTE time, including designation of an ECG technician and equipment for triage ECG, organization of triage education, improvement of 
triage disposition, and data feedback ${ }^{13}$. Although assigning a technician and ECG equipment to the conduction of triage ECG has been shown effective for reducing DTE time $8,17,23-25$, indiscriminate ECG screening without a patient interview by an experienced emergency physician has raised the concern of increasing workload among nursing staff as well as the possibility of low cost-effectiveness ${ }^{16}$. Indeed, a previous study has reported a $30 \%$ increase in ECG workload after implementation of a triage ECG program ${ }^{10}$. By combining the strategies of cardiac triage and triage ECG, Coyne et al. have shown a reduction of DTE time by $39 \%$ (i.e., from 23 to 14 minutes) and DTB by $12 \%$ (from 85 to 75 minutes). Taking into consideration the downsides of triage ECG, the current study aims at investigating the impact of cardiac triage per se on DTE time reduction.

Our cardiac triage protocol included the triage nurse's early identification of patients with a possible ischemic heart disease by labeling the patients a red warning tag that alerted the emergency medical personnel (i.e., emergency physicians, residents, or nurse practitioners) of the need for prompt historytaking and placing their medical records in a designated box for expedited management. For patients presenting with a history suggestive of coronary heart disease, prompt ECG was acquired. In this way, indiscriminate ECG screening was avoided. This approach also eliminated the necessity of assigning nursing staff, space, and ECG equipment as required for triage ECG. This is of particular clinical importance because overcrowding in the ED is a critical issue worldwide ${ }^{26,27}$ and efficient utilization of medical manpower remains one of the formidable challenges to healthcare organizations. Moreover, although the proportion of patients (8.84\%) receiving ECG in our ED in the post-intervention group was not increased compared with that in the pre-intervention group (8.83\%), our study demonstrated that the achievement rate of DTE $<10$ minutes and DTB $<90$ minutes were improved from $78.9-95 \%(20.4 \%)$ and $68.4-83.3 \%(21.8 \%)$, respectively (both $p<0.05$ ). The findings, therefore, indicate significant reductions in both DTE and DTB without increasing the ECG workload.

Furthermore, through adopting the concept of mass casualty triage ${ }^{28}$, the triage nurse labeled the patients suspected of experiencing acute coronary syndrome with a red tag and placed their medical records in a designated box to expedite medical attention by emergency clinicians in a busy and noisy environment as well as the acquisition of an ECG for early diagnosis, thereby enabling prompt primary $\mathrm{PCl}$ for confirmed cases of STEMI.

As a DTE time over 10 minutes is an indicator of unacceptable emergency medical practice ${ }^{1}$, we investigated the effectiveness of our interventions for reducing the DTE time by selecting the predictors previously reported to be related to DTE > 10 minutes, including the female gender ${ }^{9}$, STEMI without chest pain $^{10,15}$, relatively non-severe initial presentations (i.e., Triage Category III, IV, $\left.\mathrm{V}\right)^{22}$, and walk-in patients ${ }^{8}$, for analysis. Among them, DTE time of STEMI patients assigned into a low (i.e., less severe) triage category was significantly improved after intervention. The designation of triage levels to patients with cardiac ischemic symptoms by triage nurses might be affect by multiple factors, including patient's characteristics, acute myocardial infarction volume, or subjective experience of triage nurses ${ }^{22,29}$. Clare et al. has reported that up to one third of patients with STEMI could have an initial non-severe presentation 
(i.e., a low triage score), resulting in prolonged DTE and DTB times ${ }^{29}$. Albeit not as high as the proportion previously reported, there were still $17 \%$ of STEMI patients being assigned to a low triage category in our study. Although there was no significant difference in the proportion of patients with a low triage score between pre- and post-intervention groups in the current study as well as in a previous triage ECG report ${ }^{22}$, the percentage of patients with STEMI assigned with a low triage score decreased significantly from $90 \%$ (9 of 10$)$ to $10 \%$ (1 of 10$)(p<0.01)$ after cardiac triage implementation.

Despite the lack of statistical significance, DTE > 10 minutes in female gender and walk-in patients were decreased after our intervention. Female gender has been reported as a strong independent predictor of delayed ECG acquisition in several literature reviews ${ }^{12,22,30}$. Possible reasons for delayed ECG in females include atypical symptom presentation and the concern for ECG acquisition-related violation of personal privacy to which a sufficient number of female triage nurses has been reported to be a possible solution ${ }^{9}$. During the post-intervention period, all female patients undergoing cardiac triage received ECG performed by female nurse practitioners so that the influence of personal privacy on DTE time could be minimized. Additionally, the mode of arrival may also contribute to a prolonged DTE time $\mathrm{e}^{31}$. Literature review showed that patients with walk-in arrival are more likely to be designated into a low triage category compared with those arrived by ambulance ${ }^{32}$, contributing to a possible delay in receiving medical attention under the circumstances of ED overcrowding. This is supported by our study in which all STEMI patients with DTE > 10 minutes arrived at the ED on foot in both pre-and post-intervention groups. Utilizing cardiac triage with a red warning tag could expedite ECG examination for patients presenting with ischemic cardiac symptoms even if they belong to a low triage category.

There were $14 \%$ and $6 \%$ of STEMI patients without chest pain in our pre- and post-invention groups, respectively. The figure was within the range of $6-9 \%$ previously reported ${ }^{15,33}$. Our results showed no significant difference in the rate of DTE time $<10$ minutes before and after implementation of the cardiac triage program ( $37.5 \%$ vs. $50 \%$, respectively), indicating no notable benefit in this particular patient population. One of the possible reasons could be atypical initial presentations of STEMI such as general discomfort, dizziness or weakness in some of the patients, which have not been included in the AHA screening guidelines ${ }^{20}$. Further emendations of the cardiac triage protocol may be necessary to expand the criteria for inclusion. Nevertheless, the number of patients with atypical STEMI presentations was too small to arrive at a robust conclusion.

The present study had its limitations. Firstly, the statistical power and reliability of our results were limited by the relatively small number of patients, which was due to the single center nature of the current study instead of a nationwide investigation. Besides, STEMI patients usually comprise only a minor portion of patients visiting the ED during the study period. Second, because the modified cardiac triage protocol is aimed at expediting STEMI patient management in a high-volume emergency care setting as a quality improvement strategy, its feasibility and effectiveness in other ED settings remain to be validated. Third, the accuracy of data acquisition may be hampered by ambiguous symptom descriptions in medical 
records, for which experts in the quality control team were recruited as reviewers to categorize the nature of those symptoms to minimize the impact of this potential confounder.

In conclusion, utilizing a modified chief complaint-based cardiac triage strategy, the current study showed that the DTE and DTB times could both be significantly shortened for STEMI patients. Moreover, the pitfall of failure in early discrimination of patients with STEMI associated with the conventional triage system could also be improved as reflected by the shortened ECG time in STEMI patients with initially underestimated disease severity.

\section{Declarations}

\section{Data Availability}

Data relevant to the present study are available on request made to the corresponding author.

\section{Author Contributions Statement}

HYS, JLT, CKS and CWH conceived and designed the study and drafted manuscript. JLT, CSC, KHL and $\mathrm{CWH}$ conducted data extraction and manual chart review. $\mathrm{YCH}$ and YHW performed data analysis. HYS and $\mathrm{CWH}$ critically revised manuscript and final approval.

\section{Additional Information}

All authors have no commercial association, such as consultancies, stock ownership, or other equity interests or patent-licensing arrangements. The authors declare that they have no competing interests.

\section{References}

1 Antman, E. M. et al. ACC/AHA guidelines for the management of patients with ST-elevation myocardial infarction-executive summary. A report of the American College of Cardiology/American Heart Association Task Force on Practice Guidelines (Writing Committee to revise the 1999 guidelines for the management of patients with acute myocardial infarction). J Am Coll Cardiol.44, 671-719 (2004).

2 Neumann, F. J. et al. 2018 ESC/EACTS Guidelines on myocardial revascularization. Eur Heart J.40, 87165 (2019).

3 Chen, F. C., Lin, Y. R., Kung, C. T., Cheng, C. I. \& Li, C. J. The Association between Door-to-Balloon Time of Less Than 60 Minutes and Prognosis of Patients Developing ST Segment Elevation Myocardial Infarction and Undergoing Primary Percutaneous Coronary Intervention. Biomed Res Int.2017, 1910934; 10.1155/2017/1910934 (2017).

4 Kuo, F. Y. et al. The effect of failure mode and effect analysis on reducing percutaneous coronary intervention hospital door-to-balloon time and mortality in ST segment elevation myocardial infarction. 
BMJ Qual Saf.22, 626-638 (2013).

5 McCabe, J. M. et al. Impact of door-to-activation time on door-to-balloon time in primary percutaneous coronary intervention for ST-segment elevation myocardial infarctions: a report from the Activate-SF registry. Circ Cardiovasc Qual Outcomes.5, 672-679 (2012).

6 Bradley, E. H. et al. Strategies for reducing the door-to-balloon time in acute myocardial infarction. $N$ Engl J Med.355, 2308-2320 (2006).

$7 \mathrm{Krumholz}, \mathrm{H}$. M. et al. A campaign to improve the timeliness of primary percutaneous coronary intervention: Door-to-Balloon: An Alliance for Quality. JACC Cardiovasc Interv.1, 97-104 (2008).

8 Takakuwa, K. M., Burek, G. A., Estepa, A. T. \& Shofer, F. S. A method for improving arrival-toelectrocardiogram time in emergency department chest pain patients and the effect on door-to-balloon time for ST-segment elevation myocardial infarction. Acad Emerg Med.16, 921-927 (2009).

9 Keats, A. et al. A quality improvement project to reduce door-to-electrocardiogram time: A multicenter study. J Saudi Heart Assoc.30, 180-187 (2018).

10 Lee, C. K. et al. The impact of door-to-electrocardiogram time on door-to-balloon time after achieving the guideline-recommended target rate. PLoS One.14, e0222019; 10.1371/journal.pone.0222019 (2019).

11 Menees, D. S. et al. Door-to-balloon time and mortality among patients undergoing primary PCl. N Engl J Med.369, 901-909 (2013).

12 Diercks, D. B. et al. Frequency and consequences of recording an electrocardiogram $>10$ minutes after arrival in an emergency room in non-ST-segment elevation acute coronary syndromes (from the CRUSADE Initiative). Am J Cardiol.97, 437-442 (2006).

13 Chhabra, S., Eagles, D., Kwok, E. S. H. \& Perry, J. J. Interventions to reduce emergency department doorto- electrocardiogram times: A systematic review. CJEM.21, 607-617 (2019).

14 Phelan, M. P. et al. Improving emergency department door-to-electrocardiogram time in ST segment elevation myocardial infarction. Crit Pathw Cardiol.8, 119-121 (2009).

15 Borden, W. B. et al. Quality improvement in the door-to-balloon times for ST-elevation myocardial infarction patients presenting without chest pain. Catheter Cardiovasc Interv.79, 851-858 (2012).

16 Noll, S. et al. The utility of the triage electrocardiogram for the detection of ST-segment elevation myocardial infarction. Am J Emerg Med.36, 1771-1774 (2018).

17 Coyne, C. J. et al. Improving door-to-balloon time by decreasing door-to-ECG time for walk-in STEMI patients. West J Emerg Med.16, 184-189 (2015). 
18 Wiler, J. L. et al. Optimizing emergency department front-end operations. Ann Emerg Med.55, 142160.e141 (2010).

$19 \mathrm{Ng}, \mathrm{C}$. J. et al. Validation of the Taiwan triage and acuity scale: a new computerised five-level triage system. Emerg Med J.28, 1026-1031 (2011).

20 Yiadom, M. Y. et al. Performance of Emergency Department Screening Criteria for an Early ECG to Identify ST-Segment Elevation Myocardial Infarction. J Am Heart Assoc.6, e003528;

10.1161/jaha.116.003528 (2017).

21 Chen, K. C., Yen, D. H., Chen, C. D., Young, M. S. \& Yin, W. H. Effect of emergency department in-hospital tele-electrocardiographic triage and interventional cardiologist activation of the infarct team on door-toballoon times in ST-segment-elevation acute myocardial infarction. Am J Cardiol.107, 1430-1435 (2011).

22 Atzema, C. L., Austin, P. C., Tu, J. V. \& Schull, M. J. ED triage of patients with acute myocardial infarction: predictors of low acuity triage. Am J Emerg Med.28, 694-702 (2010).

23 Kaila, K. S. et al. Reperfusion times for ST elevation myocardial infarction: a prospective audit. Mcgill J Med.10, 75-80 (2007).

24 Piggott, Z., Weldon, E., Strome, T. \& Chochinov, A. Application of Lean principles to improve early cardiac care in the emergency department. CJEM.13, 325-332 (2011).

25 Sprockel, J. J. et al. Optimization of door-to-electrocardiogram time within a critical pathway for the management of acute coronary syndromes at a teaching hospital in Colombia. Crit Pathw Cardiol.14, 2530 (2015).

26 Jayaprakash, N., O'Sullivan, R., Bey, T., Ahmed, S. S. \& Lotfipour, S. Crowding and delivery of healthcare in emergency departments: the European perspective. West J Emerg Med.10, 233-239 (2009).

27 Tanaka, K. et al. Development of a novel information and communication technology system to compensate for a sudden shortage of emergency department physicians. Scand J Trauma Resusc Emerg Med.25, 6; 10.1186/s13049-017-0347-3 (2017).

28 Lee, C. H. Disaster and mass casualty triage. AMA J Ethics.12, 466-470 (2010).

29 Atzema, C. L., Schull, M. J., Austin, P. C. \& Tu, J. V. Temporal changes in emergency department triage of patients with acute myocardial infarction and the effect on outcomes. Am Heart J.162, 451-459 (2011).

30 Blomkalns, A. L. et al. Gender disparities in the diagnosis and treatment of non-ST-segment elevation acute coronary syndromes: large-scale observations from the CRUSADE (Can Rapid Risk Stratification of Unstable Angina Patients Suppress Adverse Outcomes With Early Implementation of the American 
College of Cardiology/American Heart Association Guidelines) National Quality Improvement Initiative. J Am Coll Cardiol.45, 832-837 (2005).

31 Bansal, E. et al. Importance of hospital entry: walk-in STEMI and primary percutaneous coronary intervention. West J Emerg Med.15, 81-87 (2014).

32 Canto, J. G. et al. Use of emergency medical services in acute myocardial infarction and subsequent quality of care: observations from the National Registry of Myocardial Infarction 2. Circulation.106, 30183023 (2002).

33 Björck, L. et al. Absence of chest pain and long-term mortality in patients with acute myocardial infarction. Open Heart.5, e000909; 10.1136/openhrt-2018-000909 (2018).

\section{Figures}




\section{STEMI CHECKLIST}

Chart Number:

Name of Patient:

*Time of chest pain onset:

*Time of hospital arrival (Triage):

*Time of cardiologist consultation:

*Time of cardiologist arrival:

ECG:

"FIRST" 12-lead ECG time:

Door to ECG time: $\min$

Reason for $>10$ minutes:

If inferior wall $\mathrm{MI}$, do right side ECG

\section{Medications:}

Aspirin $300 \mathrm{mg}$ PO stat

Ticagrelor $180 \mathrm{mg}$ PO stat or Clopidogrel 300-600 mg PO

$\square$ Unfractionated heparin IV bolus 60 unit/kg (Max 4000 unit stat)

$\square$ Morphine 2-4 mg IV stat

$\square$ Nitroglycerin $0.4 \mathrm{mg} \mathrm{SL}$ stat

${ }^{*}$ Cardiopulmonary resuscitation: $\square$ OHCA $\square$ IHCA

Emergency Physician:

\section{Figure 1}

Emergency department checklist for patients with ST-elevation myocardial infarction (STEMI) summarizing timings of key management and medications administered to be completed by emergency physician. ECG electrocardiogram, MI myocardial infarction, PO administration through oral route, IV intravenous, SL sublingual, OHCA out-of-hospital cardiac arrest, IHCA in-hospital cardiac arrest. 


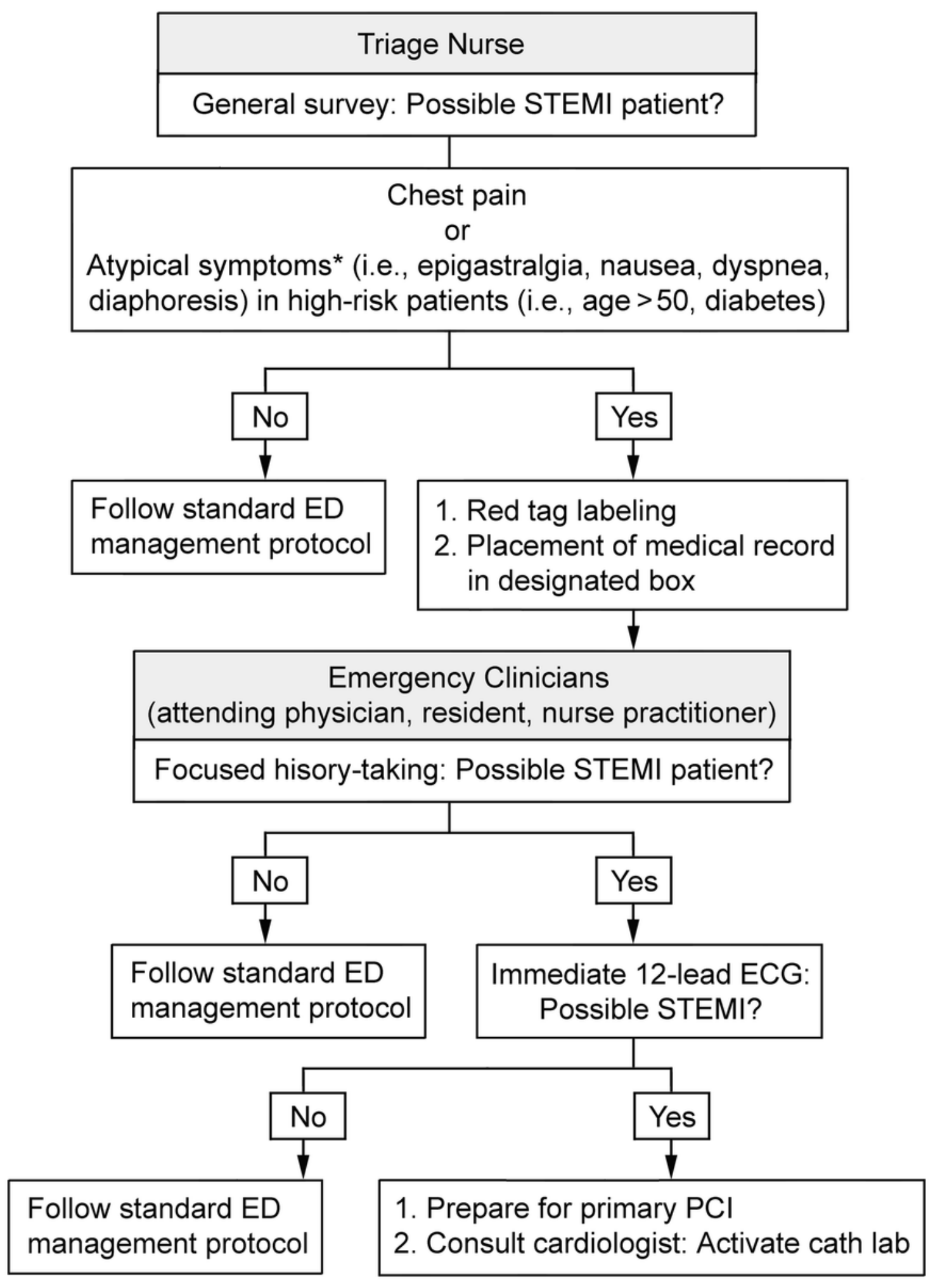

\section{Figure 2}

Modified cardiac triage strategy for expediting management of patients with possible ST-elevation myocardial infarction (STEMI). ED emergency department, PCI percutaneous coronary intervention. *Atypical symptoms according to American Heart Association emergency department screening criteria for STEMI patients. 


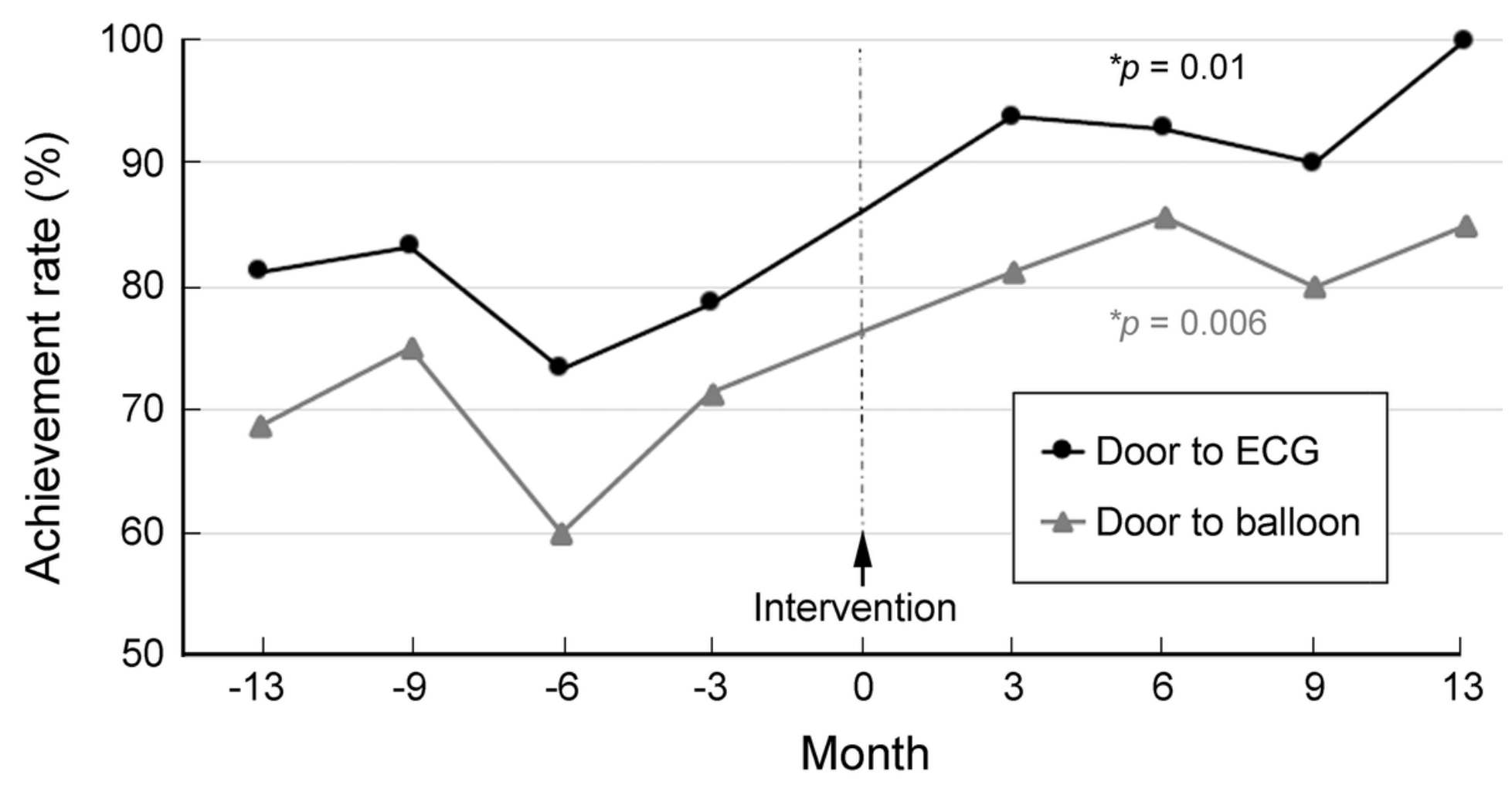

Figure 3

Changes in achievement rates of "Door to ECG" (DTE) time < 10 minutes and "Door to Balloon" (DTB) time $<90$ minutes after implementation of cardiac triage strategy. *Significance of difference determined by paired sample t-test. 